\title{
Breast Cancer Characteristics and Survival Differences between Maori, Pacific and other New Zealand Women Included in the Quality Audit Program of Breast Surgeons of Australia and New Zealand
}

\author{
Ian Campbell ${ }^{1 *}$, Nina Scott ${ }^{2}$, Sanjeewa Seneviratne ${ }^{1}$, James Kollias ${ }^{3,4}$, David \\ Walters $^{3}$, Corey Taylor ${ }^{5}$, David Roder ${ }^{6}$
}

\begin{abstract}
Background: The Quality Audit (BQA) program of the Breast Surgeons of Australia and New Zealand (NZ) collects data on early female breast cancer and its treatment. BQA data covered approximately half all early breast cancers diagnosed in NZ during roll-out of the BQA program in 1998-2010. Coverage increased progressively to about $80 \%$ by 2008 . This is the biggest $\mathrm{NZ}$ breast cancer database outside the NZ Cancer Registry and it includes cancer and clinical management data not collected by the Registry. We used these BQA data to compare socio-demographic and cancer characteristics and survivals by ethnicity. Materials and Methods: BQA data for 1998-2010 diagnoses were linked to NZ death records using the National Health Index (NHI) for linking. Live cases were followed up to December 31 ${ }^{\text {st }} \mathbf{2 0 1 0}$. Socio-demographic and invasive cancer characteristics and disease-specific survivals were compared by ethnicity. Results: Five-year survivals were $87 \%$ for Maori, $84 \%$ for Pacific, 91\% for other NZ cases and $90 \%$ overall. This compared with the $86 \%$ survival reported for all female breast cases covered by the NZ Cancer Registry which also included more advanced stages. Patterns of survival by clinical risk factors accorded with patterns expected from the scientific literature. Compared with Other cases, Maori and Pacific women were younger, came from more deprived areas, and had larger cancers with more ductal and fewer lobular histology types. Their cancers were also less likely to have a triple negative phenotype. More of the Pacific women had vascular invasion. Maori women were more likely to reside in areas more remote from regional cancer centres, whereas Pacific women generally lived closer to these centres than Other NZ cases. Conclusions: NZ BQA data indicate previously unreported differences in breast cancer biology by ethnicity. Maori and Pacific women had reduced breast cancer survival compared with Other NZ women, after adjusting for socio-demographic and cancer characteristics. The potential contributions to survival differences of variations in service access, timeliness and quality of care, need to be examined, along with effects of comorbidity and biological factors.
\end{abstract}

Keywords: Breast cancer - survival - ethnicity - risk factors - Australia and New Zealand

Asian Pac J Cancer Prev, 16 (6), 2465-2472

\section{Introduction}

Analysis of data on early invasive breast cancers diagnosed in 1998-2010, extracted from the Breast Quality Audit (BQA) database of Breast Surgeons of Australia and New Zealand (NZ), found significant inequities in survival by ethnicity. Five year survival was $87 \%$ for Maori, $84 \%$ for Pacific and $91 \%$ for Other NZ women (Campbell et al., 2014). Earlier NZ Cancer Registry data for 1994-2002 diagnoses showed a similar pattern with survivals being lower for Pacific than Maori women and highest for Other NZ women (Jeffreys et al., 2005). Other studies confirm that Maori women have had lower survivals from breast cancer than non-Maori women for successive biennial diagnostic periods since 1998-91(Soeberg et al., 2012; New Zealand Ministry of Health, 2012a).

The reasons for lower survivals for Maori and Pacific women compared with Other women are not

${ }^{I}$ Waikato Clinical School, Faculty of Medical and Health Sciences, University of Auckland, ${ }^{2}$ Chronic Conditions Advisor Te Puna Oranga, Maori Health, Waikato District Health Board, Hamilton, New Zealand, ${ }^{3}$ National Breast Cancer Audit Steering Committee, Breast Surgeons of Australia and New Zealand, Sydney, NSW; ${ }^{4}$ Breast Endocrine \& Surgical Oncology Unit, Royal Adelaide Hospital, ${ }^{5}$ Breast Surgeons of Australia and New Zealand Quality Audit, ${ }^{6}$ Cancer Epidemiology and Population Health, University of South Australia, Adelaide, Australia*For correspondence: Ian.Campbell@waikatodhb.health.nz 


\section{Ian Campbell et al}

fully understood. Differences in stages at diagnosis are contributing factors but inequities are not eliminated when survival is adjusted by characteristics associated with stage and other prognostic factors at diagnosis (Campbell et al., 2014). Inequities in rates of co-morbidity are thought to contribute, together with differences in service quality, such as access, timeliness and other quality aspects of treatment services (Rush et al., 2002; Campbell et al., 2014). The possibility of differences in cancer biology has been raised although direct evidence of such differences has not been presented. BQA data provide an opportunity to explore differences in cancer characteristics and socio-demographic differences by ethnicity and to explore inequalities in survivals after adjusting for these differences, which may point to differences in service quality.

Analysis of QA data on early invasive breast cancers diagnosed in 1998-2010, found a five-year disease-specific survival of $90 \%$ for NZ cases (Campbell et al., 2014). This survival was higher than the $86 \%$ five-year relative survival recorded for all breast cancers on the NZ Cancer Registry (New Zealand Ministry of Health, 2012b).

BQA data have been collected in NZ since 1998 and are used in this study to investigate differences in sociodemographic, patient and cancer characteristics between Maori, Pacific and Other women in NZ who presented to participating surgeons (ASERNIP-S, 2005; Wang et al., 2008; Roder et al., 2010). The main purpose of BQA data is to facilitate self-audit by surgeons but these data can also be used for research (ASERNIP-S, 2005; Wang et al., 2008). Operational details of the BQA have been described previously (ASERNIP-S, 2005; Wang et al., 2008; Roder et al., 2010). Surgeons submit their data to the BQA database and receive feedback on their clinical practices and data from all BQA surgeons in aggregate for selfauditing against defined quality indicators (ASERNIP-S, 2005; Wang et al., 2008).

BQA data have the advantage of including more clinical detail than available in the NZ Cancer Registry but they do not cover the whole population. While coverage increased to about $80 \%$ during the BQA rollout period included in this study, coverage was closer to $50 \%$ on average during this period. BQA data appear to validly represent the NZ population, however, in that they confirm differences in survival by ethnicity observed in previous studies (Jeffreys et al., 2005; New Zealand Ministry of Health, 2012a; 2012b; Soeberg et al., 2012; Campbell et al., 2014). The data also seem valid in that they confirm differences in survivals expected across conventional clinical risk factors, such as histology type, tumour size, grade, nodal status, hormone receptor status, HER-2 status, phenotype, evidence of vascular invasion, tumour location in the breast, and number of tumour foci (Campbell et al., 2014).

In this report, descriptive BQA data on patients, breast cancers and survivals are presented. Death data used for this purpose were obtained by linking BQA data to official $\mathrm{NZ}$ death records. This is a process routinely undertaken with between cancer registry and death data around the world to produce survival data, including with NZ Cancer Registry data (Jeffreys et al., 2005; Coleman et al., 2011;
Australian Institute of Health and Welfare, 2012; Cancer Research UK, 2013; Howlader et al., 2013).

Differences by ethnicity are explored that may have relevance to clinical practice and health policy, with the aim of contributing to achieving equity in access, timeliness and quality of breast cancer care and in breast cancer outcomes for all women. Approval for the study was obtained from the research ethics committee of the Royal Australasian College of Surgeons.

\section{Materials and Methods}

The study included 10,824 early invasive breast cancers diagnosed in New Zealand females between January $1^{\text {st }} 1998$ and December $31^{\text {st }} 2010$ who were treated by breast surgeons participating in the BQA. The distribution of cases by three-yearly diagnostic epoch was $11.1 \%$ prior to $2001 ; 19.5 \%$ in $2001-2003 ; 27.0 \%$ in 2004-2006; and 35.9\% in 2007-2009; plus a further $6.5 \%$ already entered for 2010. (Campbell, 2014) Death data were obtained from the NZ National Mortality Collection by linking with the BQA database through deterministic matching using the National Health Index (NHI). The NHI comprises a unique alphanumeric identifying number for each New Zealander (New Zealand Ministry of Health, 2013). Death data were traced to December $31^{\text {st }} 2010$.

The BQA data apply to early breast cancer defined similarly to local and regional spread of disease, as recorded by Surveillance, Epidemiology and End Results (SEER) Program and the NSW Cancer Registry (Roder et al., 2010; Campbell et al., 2014), although regional cancers with fixed nodes, which are regarded as regional by SEER and the NSW Registry, are not included in the Audit definition of early breast cancer (NHMRC National Breast Cancer Centre, 2001; Roder et al., 2010; Campbell et al., 2014).

BQA data included: (1) Person characteristics- age at diagnosis (yrs.), ethnicity (Maori, Pacific, Other), geographic remoteness of residence from a regional cancer centre $(0-9 \mathrm{~km}, 10-100 \mathrm{~km},>100 \mathrm{~km})$, and deprivation index of residential area (classified on an ordinal scale from 1 (least deprived) to 4 (most deprived)) (Salmond et al., 2007); (2) Tumour characteristics- histology type (ductal, lobular, other), tumour size $(\mathrm{mm})$, grade (low, intermediate, high), nodal status, oestrogen and progesterone receptor status, HER-2 receptor status, phenotype (basal (triple negative), luminal A, luminal B, ER-/PR-/HER-2+, other), vascular invasion, tumour location (ICD-O-3 classification), whether unilateral or synchronous bilateral tumour, and number of cancer foci(DeVita et al., 2008; Roder et al., 2010; Campbell et al., 2014); and (3) Other descriptors-diagnostic year, patient referral type (symptomatic, BreastScreen Aotearoa, other) and annual surgeon case load $(<10,11-30,31-100$, 101+) (Roder et al., 2010, Campbell et al., 2014).

Comparisons were made by ethnicity, first using the Pearson chi-square test for binary and nominal variables and the Kruskal-Wallis ANOVA for ordinal variables (Armitage and Berry, 1987; STATA, 2012). Multivariable logistic regression was used to derive relative odds (odds ratios) of Maori and Pacific ethnicity compared with 
Other ethnicity by descriptor. Variables were retained in multivariable models if "p values" were less than, or equal to the conventional 5\% level, or if they were considered to be of significant clinical or population health importance. Assumptions underlying regression models, including co-linearity assumptions, were tested and found to be met (Armitage and Berry, 1987; STATA, 2012).

Traditional Kaplan-Meier disease-specific survivals from breast cancer were calculated (Armitage and Berry, 1987; STATA, 2012). Breast cancer deaths were defined as those where breast cancer was recorded as the direct or antecedent cause on the death certificate, as described previously. Disease-specific survival using this definition can be a valid epidemiological option for populationbased studies when death certificate recording is of an adequate standard (Sarfati et al., 2010; Utada et al., 2012) This has been shown to give similar results to relative survival in population-based studies of breast cancer (South Australian Cancer Registry, 1999; Campbell et al., 2014). Disease-specific survival may be preferred for clinical studies where due to referral practices, patients may not be representative of the population and may have risks of alternative causes of death that do not equate with population norms. Traditional diseasespecific survival also was used in an earlier study of the Australian component of the Quality Audit database and it was considered that its use for the NZ component would assist the production of comparative data (Roder, 2010).

Survival times were calculated from date of diagnosis to December $31^{\text {st }} 2010$ or time of death, whichever came first. Hazards ratios and $95 \%$ confidence limits for breast cancer death were calculated by patient and cancer descriptors using Cox proportional hazards regression and the same censoring criteria as for the Kaplan-Meier analyses (Armitage and Berry, 1987; STATA, 2012).

Survival analyses were repeated using only cases with complete data for all variables (i.e., using "complete case analyses") and by competing risk regression (Fine, 1999). Results were very similar to those presented from the traditional Kaplan-Meier and proportional hazards regression methods and are not presented in this report. In particular, hazards ratios for variables with higher numbers of missing values (due to not being core items throughout the study period) were similar in the complete and full case analysis. Imputation of missing values was not undertaken due to the similarity of these results.

\section{Results}

\section{Socio-demographic and cancer characteristics}

Bi-variable analyses: Socio-demographic differences by ethnicity are shown in Table 1 . They include differences by: $i)$ Age at diagnosis $(\mathrm{p}<0.001)$ : younger age distributions for Maori and Pacific than women of Other ethnicity, the proportion under 40 years being $11 \%$, $13 \%$ and $7 \%$ respectively. Compared with other women, the percentage under 40 years was significantly higher for both Maori $(\mathrm{p}<0.001)$ and Pacific women $(\mathrm{p}<0.001)$. Differences between Maori and Pacific women were not statistically significant, however, neither in the percentage under 40 years $(\mathrm{p}=0.294)$ or in the age distribution overall $(\mathrm{p}=0.544)$. ii) Deprivation index $(\mathrm{p}<0.001)$ : greater proportions of Maori and Pacific than Other women ranked in the most deprived category, the percentages being $46 \%, 47 \%$ and $17 \%$ respectively. iii) Remoteness index $(\mathrm{p}<0.001)$ : a greater proportion of Maori $(34 \%)$ and lower proportion of Pacific $(6 \%)$ than Other women $(21 \%)$ were classified in the most remote residential category $(>100 \mathrm{~km}$ from a regional cancer centre).

Differences in cancer and other characteristic by ethnicity are shown in Table 2. They include differences by: iv) Histology type $(\mathrm{p}<0.001)$ : the percentage classified as ductal higher for Maori $(85 \%)(\mathrm{p}<0.001)$ and Pacific $(87 \%)(\mathrm{p}<0.001)$ than Other women $(78 \%)$. Lobular lesions comprised a low proportion of Pacific (5\%), compared with Maori $(8 \%)(\mathrm{p}=0.045)$ cases and Other cases $(12 \%)(\mathrm{p}<0.001) . v)$ Size of cancer $(\mathrm{p}<0.001)$ : larger sizes for Maori $(p<0.001)$ and Pacific $(p<0.001)$ than Other women, the proportion over $20 \mathrm{~mm}$ being $49 \%$, $59 \%$ and $41 \%$ respectively. vi) Grade $(\mathrm{p}<0.001)$ : low grade tumours were less common among Pacific women (17\%) $(\mathrm{p}<0.001)$ and although not statistically significant, among Maori cases $(23 \%)(\mathrm{p}=0.151)$ than Other women $(27 \%)$. vii) Nodal status $(\mathrm{p}<0.001)$ : node positive cancers more common in Maori (44\%) and Pacific (48\%) than Other women (38\%), viii) Oestrogen receptor $(\mathrm{p}=0.007)$ : $76 \%$ of Pacific women with oestrogen receptor positive cancers, which was lower than the $83 \%$ for Maori $(\mathrm{p}=0.002)$ and $80 \%$ for Other women $(\mathrm{p}=0.028)$.

ix) Progesterone receptor ( $\mathrm{p}=0.002)$ : $66 \%$ of Pacific women with progesterone positive cancers, which was lower than the $72 \%$ for Maori women $(\mathrm{p}=0.017)$, but not significantly different than the $68 \%$ for Other women $(\mathrm{p}=0.479) . x)$ HER-2 receptor $(\mathrm{p}<0.001: 27 \%$ of Pacific women with receptor positive cancers, which was higher than the $22 \%$ for Maori, although not reaching statistical significance $(\mathrm{p}=0.074)$, and the $16 \%$ for Other women. Compared with Other women, the percentage with receptor positive cancers was significantly higher both for Maori $(\mathrm{p}<0.001)$ and Pacific $(\mathrm{p}<0.001)$ women. $x i)$ Phenotype $(\mathrm{p}<0.001)$. Differences applied for: (triple negative) phenotype $(\mathrm{p}<0.001)$, with a higher proportion of triple negative tumours for Other ethnicity (12\%) than for Maori $(6 \%)(\mathrm{p}<0.001)$ and Pacific women $(7 \%)$ ( $\mathrm{p}=0.023)$; and ER-/PR-/HER2+ phenotype $(\mathrm{p}<0.001)$, with a higher proportion for Pacific women (17\%) being affected than Maori $(7 \%)(\mathrm{p}<0.001)$ or Other women $(6 \%)$ $(\mathrm{p}<0.001)$. A statistically significant difference in luminal A proportions was not found by ethnicity $(\mathrm{p}=0.163)$. xii) Vascular invasion $(\mathrm{p}<0.001)$, with a higher percentage showing invasion among Pacific (56\%) than Maori and Other women (both $33 \%$ ).

Differences also presented by: xiii) Referral type $(\mathrm{p}=0.004)$ : Pacific women were more likely to be referred for symptomatic reasons $(66 \%)$ than Maori $(59 \%)$ or Other women (60\%). Conversely the proportion referred from BreastScreen was lower for Pacific (19\%) than Maori (34\%) or Other women (31\%). xiv) Annual surgeon case load $(\mathrm{p}<0.001)$ : Maori women were more likely to attend low-case load surgeons ( $<30$ cases per year), the proportion being $39 \%$ compared with $19 \%$ for Pacific and $31 \%$ for Other women. 
Table 1. Socio-demographic Characteristics and Surgeon Case Loads for Maori, Pacific Islander and other NZ Women with Invasive Breast Cancer; Breast Quality Audit, 1998-2010*

\begin{tabular}{|c|c|c|c|c|c|c|}
\hline \multirow{2}{*}{$\begin{array}{l}\text { Characteristics } \\
\text { All }\end{array}$} & \multirow{2}{*}{$\begin{array}{c}\text { Case } \\
\text { Numbers }\end{array}$} & $\begin{array}{c}\text { Other } \\
\% \text { (number) }\end{array}$ & \multicolumn{2}{|c|}{$\begin{array}{c}\text { Maori } \\
\% \text { (number) }\end{array}$} & $\begin{array}{c}\text { Pacific } \\
\% \text { (number) }\end{array}$ & \multirow[t]{2}{*}{ P value* } \\
\hline & & $100 \%(9024)$ & \multicolumn{2}{|l|}{$100 \%(1428)$} & $100 \%(372)$ & \\
\hline \multicolumn{7}{|l|}{ Age (yrs.) at diag: } \\
\hline$<30$ & 65 & $0.6 \% \quad(46)$ & $1.2 \%$ & $(17)$ & $0.6 \% \quad(2)$ & $\mathrm{KWp}<0.001$ \\
\hline $30-39$ & 722 & $6.0 \% \quad(541)$ & $9.6 \%$ & $(136)$ & $12.1 \% \quad(45)$ & $\mathrm{X}^{2}{ }_{(12)} \mathrm{p}<0.001$ \\
\hline $40-49$ & 2,263 & $19.8 \%(1786)$ & $26.0 \%$ & $(370)$ & $28.8 \%(107)$ & \\
\hline $50-59$ & 2,803 & $25.1 \%(2259)$ & $31.6 \%$ & $(450)$ & $25.3 \% \quad(94)$ & \\
\hline $60-69$ & 2,677 & $25.3 \%(2275)$ & $22.5 \%$ & $(320)$ & $22.1 \% \quad(82)$ & \\
\hline $70-79$ & 1,425 & $14.2 \%(1274)$ & $8.1 \%$ & $(115)$ & $9.7 \% \quad(36)$ & \\
\hline $80+$ & 869 & $9.4 \% \quad(843)$ & $1.5 \%$ & (20) & $1.7 \% \quad(6)$ & \\
\hline \multicolumn{7}{|l|}{ Referral source: } \\
\hline Symptomatic & 4,255 & $59.6 \%(3481)$ & $59.4 \%$ & $(596)$ & $65.7 \%(178)$ & $\mathrm{X}_{(4)}^{2} \mathrm{p}=0.004$ \\
\hline BreastScreen & 2,194 & $30.8 \%(1801)$ & $34.0 \%$ & $(341)$ & $19.2 \% \quad(52)$ & \\
\hline Other & 665 & $9.6 \% \quad(558)$ & $6.6 \%$ & (66) & $15.1 \% \quad(41)$ & \\
\hline (Unknown) & $-3,710$ & -3184 & -425 & & -101 & \\
\hline \multicolumn{7}{|c|}{ Surgeon annual case load: } \\
\hline$<10$ & 715 & $6.1 \% \quad(544)$ & $11.5 \%$ & (164) & $1.9 \% \quad(7)$ & $\mathrm{X}_{(6)}^{2} \mathrm{p}<0.001$ \\
\hline 30-Nov & 2,675 & $24.7 \%(2220)$ & $27.4 \%$ & $(391)$ & $17.3 \% \quad(64)$ & $\mathrm{KWp}<0.001$ \\
\hline $31-100$ & 6,809 & $63.3 \%(5712)$ & $57.5 \%$ & $(821)$ & $74.2 \%(276)$ & \\
\hline $101+$ & 625 & $6.1 \% \quad(548)$ & $3.7 \%$ & $(52)$ & $6.8 \% \quad(25)$ & \\
\hline \multicolumn{7}{|l|}{ Deprivation index: } \\
\hline Cat 1 (least depriv.) & 1,831 & $20.5 \%(1727)$ & $6.0 \%$ & $(82)$ & $6.1 \% \quad(22)$ & $\mathrm{X}^{2}(6) \mathrm{p}<0.001$ \\
\hline Cat 2 & 2,814 & $30.0 \%(2525)$ & $16.8 \%$ & (231) & $16.2 \% \quad(58)$ & $\mathrm{KWp}<0.001$ \\
\hline Cat 3 & 3,312 & $32.9 \%(2769)$ & $31.6 \%$ & $(434)$ & $30.4 \%$ (109) & \\
\hline Cat 4 (most depriv.) & 2,201 & $16.7 \%(1403)$ & $45.7 \%$ & $(628)$ & $47.4 \%(170)$ & \\
\hline (Unknown) & -666 & -600 & -53 & & -13 & \\
\hline \multicolumn{7}{|l|}{ Remoteness index: } \\
\hline Cat $1(0-9 \mathrm{~km})$ & 3,193 & $32.8 \%(2763)$ & $20.2 \%$ & $(276)$ & $42.9 \%(154)$ & $\mathrm{X}_{(4)}^{2} \mathrm{p}<0.001$ \\
\hline Cat $2(10-100 \mathrm{~km})$ & 4,671 & $45.7 \%(3853)$ & $46.4 \%$ & $(633)$ & $51.5 \%(185)$ & $\mathrm{KWp}<0.001$ \\
\hline Cat 3 (>100) & 2,299 & $21.5 \%(1812)$ & $34.2 \%$ & $(467)$ & $5.6 \% \quad(20)$ & \\
\hline (Unknown) & -661 & -596 & -52 & & -13 & \\
\hline \multicolumn{7}{|l|}{ Diagnostic epoch: } \\
\hline $1998-2000$ & 1,201 & $11.3 \%(1018)$ & $10.3 \%$ & $(147)$ & $9.7 \% \quad(36)$ & $\mathrm{X}_{(4)}^{2} \mathrm{p}=0.090$ \\
\hline $2001-2003$ & 2,107 & $19.9 \%(1790)$ & $17.7 \%$ & $(252)$ & $17.5 \% \quad(65)$ & $\mathrm{KWp}=0.022$ \\
\hline $2004+$ & 7,516 & $68.9 \%(6216)$ & $72.1 \%$ & (1029) & $72.9 \%(271)$ & \\
\hline
\end{tabular}

$* \mathrm{KW}=$ Kruskal-Wallis ANOVA; $\mathrm{X}^{2}(\mathrm{df})=$ Pearson chi square (degrees of freedom)

There was also a tendency for more Maori and Pacific women to present in more recent diagnostic periods when compared with Other women $(\mathrm{p}=0.022)$. The number of cancer foci also varied by ethnicity $(\mathrm{p}=0.034)$, with Pacific women being more likely to have a single cancer focus.

\section{Multivariable analyses}

Maori ethnicity: Multiple logistic regression analysis indicated that the relative odds of Maori as compared with Other ethnicity (excluding Pacific women) varied according to the following characteristics:

Demographic: Younger age at diagnosis: compared with being under 30 years, the relative odds $(95 \%$ confidence limits) of Maori ethnicity reducing with increasing age to a low of $0.06(0.03,0.13)$ in women aged 80 years or more. Increase in deprivation: compared with the least deprived (category 1), the relative odds of Maori ethnicity increased to a maximum of $7.29(5.70,9.33)$ for the most deprived (category 4 ). Increase in residential remoteness: compared with the least remote of less than $10 \mathrm{~km}$ from a regional cancer centre, the relative odds of Maori ethnicity increased to $1.86(1.56,2.21)$ for the most remote category of over $100 \mathrm{~km}$ from a major centre.

Cancer: Not having a lobular histology type: compared with ductal histology, the relative odds of Maori ethnicity for lobular histology being $0.60(0.48,0.75)$. Having a larger tumour size: compared with a diameter of under $10 \mathrm{~mm}$, the relative odds of Maori ethnicity increasing with tumour size to a maximum of $3.02(2.33,3.93)$ for diameters of $40 \mathrm{~mm}$ or more. Not having a basal (triple negative) phenotype: compared with basal phenotype, the relative odds of Maori ethnicity being elevated for: luminal A at 2.39 (1.75, 3.26); luminal B at 2.92 (2.00, 4.29); and ER-/PR-/HER2+ at 2.18 (1.44, 3.31).

\section{Pacific ethnicity}

Multiple logistic regression analysis indicated that the relative odds of Pacific ethnicity as compared with Other ethnicity (excluding Maori women) varied by the following characteristics:

Demographic: Age compared with under 40 years, the relative odds of Pacific ethnicity reducing with age to 0.08 $(0.03,0.20)$ for 80 years or more. Increase in deprivation: compared with the least deprived (category 1 ), the relative 
Table 2. Cancer Characteristics of Maori, Pacific Islander and other NZ Women with Invasive Breast Cancer; Breast Quality Audit, 1998-2010*

\begin{tabular}{|c|c|c|c|c|c|}
\hline Characteristics & $\begin{array}{c}\text { Case } \\
\text { Numbers }\end{array}$ & $\begin{array}{c}\text { Other } \\
\% \text { (number) }\end{array}$ & $\begin{array}{c}\text { Maori } \\
\% \text { (number) }\end{array}$ & $\begin{array}{c}\text { Pacific } \\
\% \text { (number) }\end{array}$ & $\mathrm{P}$ value* \\
\hline \multicolumn{6}{|l|}{ Histology: } \\
\hline Ductal & 8,370 & $78.0 \%(6867)$ & $84.9 \%$ (1189) & $86.5 \%(314)$ & \multirow{4}{*}{$\mathrm{X}_{(4)}^{2} \mathrm{p}<0.001$} \\
\hline Lobular & 1,151 & $11.7 \%(1026)$ & $7.7 \% \quad(108)$ & $4.7 \% \quad(17)$ & \\
\hline Other & 1,046 & $10.3 \%(910)$ & $7.4 \% \quad(104)$ & $8.8 \% \quad(32)$ & \\
\hline (Unknown) & -257 & -221 & -27 & -9 & \\
\hline \multicolumn{6}{|l|}{$\operatorname{Size}(\mathrm{mm})$} \\
\hline \multicolumn{6}{|l|}{ Cat.1: } \\
\hline$<10$ & 1,623 & $16.0 \%(1443)$ & $10.0 \% \quad(142)$ & $10.3 \% \quad(38)$ & \multirow{6}{*}{$\begin{array}{l}\mathrm{X}^{2}{ }_{(8)} \mathrm{p}<0.001 \\
\mathrm{KW} p<0.001\end{array}$} \\
\hline $10-14$ & 1,945 & $18.6 \%(1676)$ & $16.2 \% \quad(230)$ & $10.5 \% \quad(39)$ & \\
\hline $15-19$ & 1,941 & $18.2 \%(1638)$ & $17.3 \% \quad(247)$ & $15.1 \% \quad(56)$ & \\
\hline $20-29$ & 2,644 & $24.2 \%(2181)$ & $27.2 \% \quad(388)$ & $20.2 \% \quad(75)$ & \\
\hline $30-39$ & 1,275 & $11.4 \%(1028)$ & $13.8 \% \quad(196)$ & $13.8 \% \quad(51)$ & \\
\hline $40+$ & 1,396 & $11.8 \%(1058)$ & $15.8 \% \quad(225)$ & $30.4 \%(113)$ & \\
\hline \multicolumn{6}{|l|}{ Cat.2: } \\
\hline$<20$ & 6,203 & $59.0 \%(5321)$ & $51.1 \% \quad(729)$ & $41.2 \%(153)$ & \multirow{3}{*}{$\begin{array}{l}\mathrm{X}^{2}{ }_{44} \mathrm{p}<0.001 \\
\mathrm{KW} \mathrm{p}<0.001\end{array}$} \\
\hline $21-50$ & 3,942 & $35.4 \%(3191)$ & $41.9 \% \quad(597)$ & $41.4 \%$ (154) & \\
\hline $51+$ & 679 & $5.7 \% \quad(512)$ & $7.2 \% \quad(102)$ & $17.5 \% \quad(65)$ & \\
\hline \multicolumn{6}{|l|}{ Grade: } \\
\hline Low & 2,679 & $26.6 \%(2302)$ & $22.8 \%(315)$ & $17.4 \% \quad(62)$ & \multirow{3}{*}{$\begin{array}{l}\mathrm{X}^{2}{ }_{44} \mathrm{p}<0.001 \\
\mathrm{KW} p<0.001\end{array}$} \\
\hline Intermediate & 4,515 & $42.8 \%(3705)$ & $47.1 \%(650)$ & $44.8 \%(160)$ & \\
\hline High & 3,197 & $30.6 \%(2647)$ & $30.1 \%(415)$ & $37.8 \%(135)$ & \\
\hline (Unknown) & -433 & -370 & -48 & -15 & \\
\hline \multicolumn{6}{|l|}{ Nodal status: } \\
\hline Negative & 6,483 & $62.5 \%(5515)$ & $56.1 \% \quad(776)$ & $52.5 \%(192)$ & $\mathrm{X}^{2}{ }_{2} \mathrm{p}<0.001$ \\
\hline Positive & 4,091 & $37.5 \%(3310)$ & $43.9 \% \quad(607)$ & $47.5 \%(174)$ & \\
\hline (Unknown) & -250 & -199 & -45 & -6 & \\
\hline Oestrogen recept: & & & & & \\
\hline Positive & 8,428 & $80.4 \%(7002)$ & $82.8 \%(1155)$ & $75.7 \%(271)$ & $\mathrm{X}^{2}{ }_{20} \mathrm{p}=0.007$ \\
\hline Negative & 2,033 & $19.6 \%(1706)$ & $17.2 \%(240)$ & $24.3 \% \quad(87)$ & \\
\hline (Unknown) & -363 & -316 & -33 & -14 & \\
\hline Progest. recept: & & & & & \\
\hline Positive & 7,060 & $67.8 \%(5831)$ & $72.4 \% \quad(996)$ & $66.0 \%(233)$ & $\mathrm{X}^{2}{ }_{(2)} \mathrm{p}=0.002$ \\
\hline Negative & 3,268 & $32.2 \%(2769)$ & $27.6 \% \quad(379)$ & $34.0 \%(120)$ & \\
\hline (Unknown) & -496 & -424 & -53 & -19 & \\
\hline HER-2recept**: & & & & & \\
\hline Positive & 1,052 & $16.2 \% \quad(793)$ & $21.6 \% \quad(195)$ & $27.1 \% \quad(64)$ & $\mathrm{X}^{2}{ }_{(2)} \mathrm{p}<0.001$ \\
\hline Negative & 4,977 & $83.8 \%(4099)$ & $78.4 \% \quad(706)$ & $72.9 \%(172)$ & \\
\hline (Unknown) & -4795 & -4132 & -527 & -136 & \\
\hline Phenotype**: & & & & & \\
\hline Basal (triple neg.) & 672 & $12.5 \% \quad(602)$ & $6.0 \% \quad(53)$ & $7.4 \% \quad(17)$ & $\mathrm{X}^{2}{ }^{\mathrm{p}} \mathrm{p}<0.001$ \\
\hline LuminalA & 3,599 & $60.2 \%(2910)$ & $63.1 \% \quad(561)$ & $55.9 \%(128)$ & \\
\hline LuminalB & 457 & $7.1 \% \quad(344)$ & $10.8 \%$ & $7.4 \% \quad(17)$ & \\
\hline ER-/PR-/HER2+ & 368 & $5.6 \% \quad(268)$ & $7.0 \% \quad(62)$ & $16.6 \%(38)$ & \\
\hline Other & 855 & $14.7 \% \quad(709)$ & $13.2 \%$ & $12.7 \% \quad(29)$ & \\
\hline (Unknown) & -4873 & -4191 & -539 & -143 & \\
\hline Vascularinvas.**: & & & & & \\
\hline Absent & 5,593 & $67.3 \%(4721)$ & $67.0 \%(786)$ & $44.1 \% \quad(86)$ & $\mathrm{X}^{2}{ }^{2} \mathrm{p}<0.001$ \\
\hline Present & 2,788 & $32.7 \%(2292)$ & $33.0 \%(387)$ & $55.9 \%(109)$ & \\
\hline (Unknown) & -2443 & -2011 & -255 & -177 & \\
\hline Tumourlocation $* *$ : & & & & & \\
\hline Lateral & 805 & $9.7 \% \quad(686)$ & $9.2 \% \quad(101)$ & $7.4 \% \quad(18)$ & $\mathrm{X}^{2}{ }_{(20)} \mathrm{p}=0.502$ \\
\hline Medial & 310 & $3.7 \% \quad(258)$ & $4.0 \% \quad(44)$ & $3.3 \% \quad(8)$ & \\
\hline Superior & 821 & $9.7 \% \quad(681)$ & $10.2 \% \quad(112)$ & $11.4 \% \quad(28)$ & \\
\hline Inferior & 369 & $4.5 \% \quad(314)$ & $4.4 \% \quad(49)$ & $2.5 \% \quad(6)$ & \\
\hline Central & 672 & $7.9 \% \quad(556)$ & $8.3 \% \quad(91)$ & $10.2 \% \quad(25)$ & \\
\hline Supero-lateral & 3,010 & $36.2 \%(2549)$ & $33.8 \% \quad(373)$ & $35.9 \% \quad(88)$ & \\
\hline Supero-medial & 1,022 & $12.2 \% \quad(857)$ & $11.8 \% \quad(130)$ & $14.3 \% \quad(35)$ & \\
\hline Infero-lateral & 701 & $8.1 \% \quad(572)$ & $9.6 \% \quad(106)$ & $9.4 \% \quad(23)$ & \\
\hline Infero-medial & 479 & $5.7 \% \quad(401)$ & $6.1 \% \quad(67)$ & $4.5 \% \quad(11)$ & \\
\hline Axillarytail & 92 & $1.2 \% \quad(82)$ & $0.8 \%$ & $0.4 \% \quad(1)$ & \\
\hline Multi-quadrant & 108 & $1.2 \% \quad(85)$ & $1.9 \% \quad(21)$ & $0.8 \% \quad(2)$ & \\
\hline (Unknown) & $-2,435$ & -1983 & -325 & -127 & \\
\hline Bilateral(synch): & & & & & \\
\hline No & 9,801 & $98.3 \%(8158)$ & $97.9 \%(1331)$ & $98.4 \%(312)$ & $\mathrm{X}^{2}{ }_{(2)} \mathrm{p}=0.624$ \\
\hline Yes & 174 & $1.7 \% \quad(141)$ & $2.1 \% \quad(28)$ & $1.6 \% \quad(5)$ & \\
\hline (Unknown) & -849 & -725 & -69 & -55 & \\
\hline No.ofcancers**: & & & & & \\
\hline 1 & 6,934 & $80.6 \%(5787)$ & $80.3 \% \quad(922)$ & $76.8 \%(225)$ & $\mathrm{X}^{2} \mathrm{p}=0.034$ \\
\hline 2 & 755 & $8.4 \% \quad(602)$ & $10.3 \% \quad(118)$ & $12.0 \% \quad(35)$ & $\mathrm{KWp}=0.342$ \\
\hline $3+$ & 930 & $11.0 \% \quad(789)$ & $9.4 \% \quad(108)$ & $11.3 \% \quad(33)$ & \\
\hline (Unknown) & -2205 & -1846 & -280 & -79 & \\
\hline
\end{tabular}

$* \mathrm{KW}=$ Kruskal-Wallis ANOVA; $\mathrm{X}^{2}(\mathrm{df})=$ Pearson chi square (degrees of freedom); **Missing values elevated as not core item throughout 
Ian Campbell et al

Table 3. Hazards Ratios for Death from Breast Cancer Among New Zealand women with Invasive Breast Cancer; Breast Quality Audit, 1998-2010*

\begin{tabular}{|c|c|c|c|c|}
\hline \multirow{2}{*}{ Case category } & & \multicolumn{3}{|c|}{ Multivariable proportional hazards regression } \\
\hline & & Case numbers & $\begin{array}{c}\text { Unadjusted hazards ratios } \\
{[95 \% \text { CLs }]}\end{array}$ & $\begin{array}{c}\text { Adjusted hazards ratios } \\
\text { [95\% CLs] }\end{array}$ \\
\hline \multirow[t]{7}{*}{ Age (yrs.) at diag: } & $<30$ & 65 & 1 & 1 \\
\hline & $30-39$ & 722 & $0.66[0.38,1.12]$ & $0.64[0.37,1.10]$ \\
\hline & $40-49$ & 2,263 & $0.40[0.24,0.68]$ & $0.53[0.31,0.89]$ \\
\hline & $50-59$ & 2,803 & $0.34[0.20,0.56]$ & $0.58[0.34,0.98]$ \\
\hline & $60-69$ & 2,677 & $0.36[0.22,0.61]$ & $0.68[0.4,1.15]$ \\
\hline & $70-79$ & 1,425 & $0.61[0.36,1.03]$ & $0.94[0.55,1.59]$ \\
\hline & $80+$ & 869 & $0.88[0.52,1.50]$ & $1.36[0.79,2.34]$ \\
\hline \multirow[t]{4}{*}{ Histology: } & Ductal & 8,370 & 1 & 1 \\
\hline & Lobular & 1,151 & $0.68[0.54,0.86]$ & $0.73[0.58,0.94]$ \\
\hline & Other & 1,046 & $0.61[0.47,0.78]$ & $0.75[0.58,0.97]$ \\
\hline & (Unknown) & -257 & $(1.23[0.89,1.69])$ & $(0.79[0.52,1.21])$ \\
\hline \multirow[t]{6}{*}{ Size (mm): } & $<9$ & 1,623 & 1 & 1 \\
\hline & $10-14$ & 1,945 & $1.74[1.19,2.55]$ & $1.46[1,2.15]$ \\
\hline & $15-19$ & 1,941 & $2.67[1.86,3.83]$ & $1.56[1.08,2.25]$ \\
\hline & $20-29$ & 2,644 & $4.36[3.11,6.10]$ & $2.11[1.49,2.98]$ \\
\hline & $30-39$ & 1,275 & $7.13[5.05,10.05]$ & $2.68[1.87,3.83]$ \\
\hline & $40+$ & 1,396 & $11.66[8.37,16.26]$ & $4.06[2.86,5.77]$ \\
\hline \multirow[t]{4}{*}{ Grade: } & Low & 2,679 & 1 & 1 \\
\hline & Intermediate & 4,515 & $4.18[3.13,5.6]$ & $2.63[1.96,3.53]$ \\
\hline & High & 3,197 & $10.09[7.6,13.41]$ & $4.40[3.26,5.92]$ \\
\hline & (Unknown) & -433 & $(7.03[4.89,10.1])$ & $(4.60[3.03,6.98])$ \\
\hline \multirow[t]{3}{*}{ Nodal status: } & Negative & 6,483 & 1 & 1 \\
\hline & Positive & 4,091 & $3.51[3.08,4.01]$ & $2.17[1.87,2.51]$ \\
\hline & (Unknown) & -250 & $(2.94[2.12,4.09])$ & $(2.57[1.83,3.62])$ \\
\hline \multirow[t]{6}{*}{ Phenotype: } & Basal (triple neg.) & 672 & 1 & 1 \\
\hline & Luminal A & 3,599 & $0.18[0.14,0.24]$ & $0.30[0.22,0.40]$ \\
\hline & Luminal B & 457 & $0.40[0.26,0.61]$ & $0.45[0.29,0.68]$ \\
\hline & ER-/PR-/HER2+ & 368 & $1.02[0.73,1.42]$ & $0.84[0.60,1.18]$ \\
\hline & Other & 855 & $0.60[0.45,0.82]$ & $0.76[0.56,1.03]$ \\
\hline & (Unknown) & $-4,873$ & $(0.55[0.44,0.69])$ & $(0.63[0.48,0.84])$ \\
\hline \multirow[t]{3}{*}{ Vascular invasion: } & Absent & 5,593 & 1 & 1 \\
\hline & Present & 2,788 & $3.59[3.10,4.16]$ & $1.54[1.31,1.81]$ \\
\hline & (Unknown) & $-2,443$ & $(1.98[1.67,2.34])$ & $(1.47[1.22,1.76])$ \\
\hline \multirow{6}{*}{ Tumour location: } & Other & 6,495 & 1 & 1 \\
\hline & Central & 672 & $1.36[0.99,1.87]$ & $1.40[1.02,1.92]$ \\
\hline & Supero-medial & 1,022 & $1.21[0.99,1.47]$ & $1.47[1.20,1.79]$ \\
\hline & Axillary tail & 92 & $1.21[0.91,1.60]$ & $1.39[1.05,1.84]$ \\
\hline & Multi-quadrant & 108 & $1.82[1.18,2.82]$ & $2.12[1.38,3.28]$ \\
\hline & (Unknown) & $-2,435$ & $(3.06[2.54,3.72])$ & $(1.06[0.88,1.29])$ \\
\hline \multirow[t]{4}{*}{ No. of cancers: } & 1 & 6,934 & 1 & 1 \\
\hline & 2 & 755 & $1.03[0.81,1.32]$ & $0.95[0.74,1.21]$ \\
\hline & $3+$ & 930 & $1.92[1.61,2.30]$ & $1.45[1.20,1.75]$ \\
\hline & (Unknown) & $-2,205$ & $(1.30[1.11,1.53])$ & $(1.12[0.93,1.34])$ \\
\hline \multirow[t]{4}{*}{ Referral source: } & Symptomatic & 4,255 & 1 & 1 \\
\hline & BreastScreen & 2,194 & $0.29[0.21,0.38]$ & $0.76[0.56,1.04]$ \\
\hline & Other & 665 & $0.44[0.30,0.64]$ & $0.91[0.62,1.34]$ \\
\hline & (Unknown) & $-3,710$ & $(1.10[0.96,1.26])$ & $(1.36[1.1,1.69])$ \\
\hline \multirow[t]{5}{*}{ Deprivation index: } & Cat 1 (least depriv.) & 1,831 & 1 & 1 \\
\hline & Cat 2 & 2,814 & $1.20[0.98,1.47]$ & $1.11[0.91,1.36]$ \\
\hline & Cat 3 & 3,312 & $1.34[1.11,1.63]$ & $1.33[1.09,1.62]$ \\
\hline & Cat 4 (most depriv.) & 2,201 & $1.26[1.03,1.56]$ & $1.09[0.87,1.35]$ \\
\hline & (Unknown) & -666 & $(1.33[0.97,1.82])$ & $(1.34[0.98,1.84])$ \\
\hline \multirow{3}{*}{ Ethnicity: } & Other & 9024 & 1 & 1 \\
\hline & Maori & 1428 & $1.39[1.18,1.64]$ & $1.46[1.22,1.75]$ \\
\hline & Pacific & 372 & $1.79[1.03,3.10]$ & $1.25[0.94,1.68]$ \\
\hline
\end{tabular}

*Disease-specific proportional hazards regression; live cases censored, 31/12/2010.

odds of Pacific ethnicity increasing to a maximum of 11.22 (7.07, 17.80) for the most deprived (category 4). Less residential remoteness: compared with the least remote of less than $10 \mathrm{~km}$ from a regional cancer centre, the relative odds of Pacific ethnicity decreasing to $0.23(0.14,0.37)$ for the most remote category of over $100 \mathrm{~km}$ from a centre. 
Cancer characteristics: Not having a lobular histology type: compared with ductal, the relative odds of Pacific ethnicity for lobular histology being $0.32(0.19,0.54)$. Having a larger tumour size: compared with a diameter of under $10 \mathrm{~mm}$, the relative odds of Pacific ethnicity increasing with tumour size to reach a maximum of 5.41 $(3.46,8.45)$ for diameters of $40 \mathrm{~mm}$ or more. Having vascular invasion: the relative odds of Pacific ethnicity being higher at $1.40(1.01,1.96)$ where vascular invasion was reported. Not having a basal (triple negative) phenotype: compared with basal phenotype, the relative odds of Pacific ethnicity being elevated for: luminal $\mathrm{A}$ at $2.54(1.47,4.38)$; luminal B at $2.07(1.00,4.28)$, and ER-/ PR-/HER2+ at $4.88(2.60,9.19)$.

\section{Survival}

Survival was $90 \%$ at 5 years from diagnosis and $84 \%$ at 10 years. Differences by patient and cancer characteristic, referral type and surgeon case load are shown in Table 3. Adjusted hazards ratios (95\% confidence limits) indicated the following characteristics to be predictive of breast cancer death: very young and old age; ductal histology type; larger tumour size; higher grade; positive nodal status; basal (triple negative) rather than luminal A or B phenotype; breast location of the cancer in central, superomedial, axillary tail or multi-quadrants rather than other specified locations; and cancers with three or more foci. Evidence was suggestive of higher breast cancer death rates in more deprived areas but the trend was inconsistent (Table 3). Similarly BreastScreen referrals tended to have a lower risk of breast cancer death after adjusting for diameter, nodal status, grade and other variables in the model, but statistical significance was not achieved $[\mathrm{HR}=0.76(0.56,1.04)]$.

After adjusting for all variables in the model, Maori cases had a higher death rate than Other cases [HR $=1.46$ $(1.22,1.75)]$. The death rate was also higher for Pacific than Other women, although statistical significance was not achieved $[\mathrm{HR}=1.25(0.94,1.68)]$.

\section{Discussion}

The survivals indicated by NZ BQA data accord with expected patterns by conventional cancer risk factors, which is indicative of validity (DeVita et al., 2008; Roder et al., 2010; Campbell et al., 2014) The overall five-year survival of $90 \%$ for BQA cases is higher than the $86 \%$ relative survival recorded for all NZ breast cancers by the NZ Cancer Registry (New Zealand Ministry of Health, 2012b). Although differences in statistical methodology may have contributed, this is not thought to have been a major source of bias, given evidence of similar results from these methodologies in past comparisons (South Australian Cancer Registry, 1999; South Australian Cancer Registry, 2007). The difference in survival found between the BQA cases and all NZ breast cancers is consistent with the BQA focus on early breast cancer.

The pattern of a higher five-year survival for Other women $(91 \%)$ than Maori $(87 \%)$ and Pacific (84\%) women corresponds with the pattern seen in population-based data (New Zealand Ministry of Health, 2012b). The differences by ethnicity were smaller for BQA data than reported from population-based data, potentially reflecting the BQA focus on early breast cancers and selective exclusion of more advanced cases. It is noteworthy however that lower survivals still persisted in Maori and Pacific women, despite these selection influences. Five years is also short follow up for breast cancer survival, so that these differences are likely to be considerably greater in absolute terms at 10 and 15 years.

Many differences were found between the sociodemographic and cancer characteristics of Maori, Pacific and Other NZ breast cancer cases. Some of these are well known, for example, Pacific and Maori women tended to be younger and to reside in more socioeconomically deprived areas. Also compared with other women, they were more likely to have larger cancers. This is the first time that Maori and Pacific women have been shown to have more ductal as opposed to lobular cancers, and to be less likely to have triple negative cancers. Both Maori and Pacific women had more HER2 +ve breast cancers, and Pacific women also showed an elevated proportion of tumours with vascular invasion. Higher proportions of HER2 + ve cancers in Maori and Pacific compared with Other women have been reported based on the NZ Cancer Registry data (New Zealand Ministry of Health, 2007).

It would be expected that Pacific and Maori women may tend to have higher survivals due to their lower proportions of triple negative lesions. By comparison, their survivals may tend to be lower due to larger cancers at diagnosis and an elevated ratio of ductal to lobular and other cancers. After adjusting for these variables and other predictors of breast cancer survival such as age, tumour grade, nodal status, other phenotypes, tumour location in the breast, numbers of cancer foci, and extent of socioeconomic deprivation, Maori women still had an elevated risk of breast cancer death compared with Other women. While the risk also appeared to be elevated for Pacific women in the unadjusted analysis, statistical significance was not achieved after adjustment.

The reason for the elevated hazards ratio for Maori women after adjustment for recorded risk factors is not known, although higher levels of diabetes and other co-morbidity, and more limited access to screening and treatment services, may have contributed (Rush et al., 2002; Campbell et al., 2014). Also, despite entering deprivation index scores and residential remoteness into the multi-variable survival analyses, residual confounding could have occurred, given the bluntness of these measures (Salmond et al., 2007)

Australian BQA data indicated a 5-year survival of 93\% for early breast cancer, which was higher than the $90 \%$ figure for New Zealand (Roder et al., 2010) The use of the NHI for linkage of NZ BQA data to death records would have been more exact than the probabilistic matching used with incomplete identifiers for the Australian BQA data, which likely would have contributed to an artificially higher survival for Australian than New Zealand BQA cases. However, some of the survival difference may be real due to the large Maori and Pacific populations in NZ, who have lower breast cancer survivals, and the slower access to new drugs in NZ in the last decade. 


\section{Ian Campbell et al}

There have also been questions about treatment delays in NZ. Additional study is needed to better identify factors responsible for this survival difference.

This report indicates that BQA data are epidemiologically useful data. Further augmentation of these data through data linkage would enable a broader assessment of reasons for differences in survival outcomes by ethnicity, including potential contributions from social inequality, other socio-demographic and environmental factors, differences in access to screening and treatment services, and potentially contributions from biological factors including genetic differences.

In conclusion, the survivals indicated by this study suggest a significantly lower breast cancer survival in NZ compared with Australia. This needs further exploration to determine the contribution made by methodological versus real effects. BQA data indicate previously unreported differences in breast cancer biology by ethnicity and worse survival for Maori and potentially Pacific women compared with Other NZ ethnicity. The potential contributions to these differences of social inequality, service access, timeliness and quality of care, co-morbidity and biological factors need further investigation.

\section{Acknowledgements}

Authors are indebted to NZ surgeons for the ongoing provision of the clinical data used in this study, the NZ Ministry of Health for the linkage of Audit data with NZ death records, and the NZ Cancer Society for the funding support that made this study possible. Cancer Australia is also acknowledged for providing administrative and technical staff support.

\section{References}

Armitage P, Berry G (1987). Statistical methods in medical research. Oxford, Blackwell Scientific Publications.

ASERNIP-S (2005). The National Breast Cancer Audit. Process for Managing Outliers In 'Breast Cancer Surgery'. Adelaide, Royal Australasian College of Surgeons.

Australian Institute of Health and Welfare and Australasian Association of Cancer Registries (2012). Cancer in Australia: an overview, 2012. Cancer series no.74. Cat. No. CAN 70. Canberra, Australian Institute of Health and Welfare.

Campbell ID, Scott N, Seneviratne S, et al (2014). Breast cancer survival in New Zealand women. ANZ J Surg. [In press]

Cancer Research UK (2013). Cancer survival statistics. London, Cancer research UK.

Coleman MP, Forman D, Bryant H, et al (2011). Cancer survival in Australia, Canada, Denmark, Norway, Sweden, and the UK, 1995-2007 (the International cancer benchmarking partnership): an analysis of population-based cancer registry data. Lancet, 377, 127-38.

DeVita VT, Lawrence TS, Rosenberg SA, DePinho RA, Weinberg RA (2008). Cancer: Principles and Practice of Oncology. 8th Edition. Philadelphia, Lippincott, Williams and Wilkins.

Fine JP, Gray RJ (1999). A proportional hazards model for the subdistribution of a competing risk. J Am Stat Assoc, 94, 496-509.

Howlader N, Noone AM, Krapcho M, et al (2013). SEER cancer statistics review, 1975-2010. Bethesda MD, National Cancer
Institute.

Jeffreys M, Stevanovic V, Tobias M, et al (2005). Ethnic inequalities in cancer survival in New Zealand: linkage study. Am J Public Health, 95, 834-7.

New Zealand Ministry of Health (2007). Epidemiology of HER2 positive breast cancer in New Zealand. Wellington, Ministry of Health.. http://www.pharmac.govt.nz/2007/06/13/290607c. $\mathrm{pdf} / \mathrm{text}$

New Zealand Ministry of Health (2012a). Cancer patient survival. Change over time update: covering the period 1994 to 2009. Wellington, Ministry of Health.

New Zealand Ministry of Health (2012b). Cancer: New Registrations and Deaths 2009. Wellington, Ministry of Health.

New Zealand Ministry of Health (2013). National Health Index. Wellington, Ministry of Health.

NHMRC National Breast Cancer Centre (2001). Clinical practice guidelines for the management of early breast cancer: Second edition. Canberra, NHMRC.

Roder D, Wang JX, Zorbas H, Kollias J, Maddern G (2010). Survival from breast cancers managed by surgeons participating in the national breast cancer audit of the royal Australasian college of surgeons. ANZ J Surg, 80, 776-80.

Rush EC, Plank LD, Mitchelson E, Laulau MS (2002). Central obesity and risk for type 2 diabetes in Maori, Pacific, and European young men in New Zealand. Food Nutr Bull, 23, 82-6.

Salmond C, Crampton P, Atkinson J (2007). NZDep2006 Index of Deprivation. Wellington, Department of Public Health, University of Otago.

Sarfati D, Blakely T, Pearce N (2010). Measuring cancer survival in populations: relative survivals vs cancer-specific survival. Int J Epidemiol, 39, 598-610.

Soeberg M, Blakely T, Sarfati D, et al (2012). Cancer trends: Trends in cancer survival by ethnic and socio-economic group. New Zealand 1991-2004. wellington, university of otago and ministry of health.

South Australian Cancer Registry (1999). Epidemiology of cancer in South Australia. incidence, mortality and survival 1977 to 1996 . Incidence and mortality 1996. Adelaide, Openbook Publ.

South Australian Cancer Registry (2007). Cancer in South Australia 2004, with incidence projections to 2007. Adelaide, Department of Health.

STATA (2012). Data analysis and statistical software. Version 12. college station, Texas: StataCorp LP.

Utada M, Ohno Y, Shimizu S, et al (2012). Comparison between overall, cause-specific, and relative survival rates based on data from a population-based cancer registry. Asian Pac J Cancer Prev, 13, 5681-5.

Wang J, Boult M, Roder D, et al (2008). Commentary: how surgical audits can be used to promote uptake of surgical evidence. ANZ J Surg, 78, 437-8. 